\title{
Stability Bound Analysis and Synthesis for Singularly Perturbed Systems with Time-Varying Delay
}

\author{
Fengqi Sun, ${ }^{1}$ Linna Zhou, ${ }^{2}$ Qingling Zhang, ${ }^{1}$ and Yongxiang Shen ${ }^{3}$ \\ ${ }^{1}$ Institute of Systems Science, Northeastern University, Shenyang 110819, China \\ ${ }^{2}$ School of Information and Electrical Engineering, China University of Mining and Technology, Xuzhou 221116, China \\ ${ }^{3}$ Mathematics Department, Jilin Normal University, Siping 136000, China \\ Correspondence should be addressed to Fengqi Sun; jlsdsfq@163.com
}

Received 22 September 2012; Revised 25 December 2012; Accepted 29 December 2012

Academic Editor: Baocang Ding

Copyright (C) 2013 Fengqi Sun et al. This is an open access article distributed under the Creative Commons Attribution License, which permits unrestricted use, distribution, and reproduction in any medium, provided the original work is properly cited.

\begin{abstract}
This paper addresses the problems of stability bound analysis and synthesis for singularly perturbed systems with time-varying delay. First, by constructing an appropriate Lyapunov-Krasovskii functional, a sufficient condition is derived for the system to be stable when the singular perturbation parameter is lower than a predefined upper bound which is referred to as the stability bound of the singularly perturbed system. The proposed criterion needs less computational cost than the existing ones. Then, a state feedback controller design method is proposed to achieve a prescribed stability bound, which can be applied to both standard and nonstandard singularly perturbed systems with time-varying delay. Finally, the effectiveness and merits of the proposed approach are shown through numerical examples.
\end{abstract}

\section{Introduction}

Singularly perturbed systems (SPSs) have attracted much attention due to their extensive applications in chemical processes, robots, aerospace engineering, power systems, magnetic-ball suspension systems, and so forth. Consequently, a vast amount of effort has been devoted to deriving stability criteria for SPSs with constant or time-varying delays. Therefore, the relevant theory and application research have attracted much attention [1]. Stability problem for SPSs is known as stability bound problem, which is referred to as the problem of determining the stability bound $\bar{\varepsilon}$ such that the system is stable for $\forall \varepsilon \in(0, \bar{\varepsilon}]$. Many results for stability bound problem of SPSs have been reported $[2,3]$.

Delay is a widespread physical phenomenon in practical systems $[4,5]$. In [6-10], by a reduction technique, some significant advances have been achieved in analysis and synthesis of SPSs where delays are proportional to perturbed parameter. For a more general case in which time delay and perturbed parameter are independent, there are two main classes of approaches: frequency domain techniques and LyapunovKrasovskii-functional-based approaches. In [11-14], frequency domain techniques were applied to establish stability criteria for SPSs with time delay in slow state. LyapunovKrasovskii-functional-based approaches which are usually expressed in terms of linear matrix inequalities (LMIs) are popular in recent years because LMI conditions can be easily verified by using convex optimization algorithms $[2,15]$. In [16], robust stability of uncertain SPSs with state delays has been studied by Lyapunov-Krasovskii stability theory and LMI technique. In [17], a delay-dependent LMI criterion for the stability of singularly perturbed differential-difference systems has been derived. In [18], a stability criterion was proposed for uncertain SPSs with time delays. Based on the state space approach, the exact stability bound for discretetime SPSs with multiple time delays was studied in [19]. In [20], the problem of stabilization for SPSs with multiple time delays was considered. Instead of applying the Lyapunov theory, the well-known comparison theorem and matrix measure are employed to investigate the problem. 
It should be emphasized that most of the reported results on SPSs with time delay require the time delay to be time invariant [6-20]. Little works deal with the stability problem of SPSs with time-varying delays. In [22], a stability criterion was proposed for SPSs with time-varying delay by transforming the system into an equivalent descriptor system. But the proposed results cannot produce the stability bound of SPSs. In [23], an approach to estimate the stability bound of SPSs with time-varying delays was proposed under model transformation. However, this constructive method is not easy to perform. Stability bound problem of SPSs with time-varying delay was considered in [21] and an LMI-based method was proposed, which is easy to be solved by LMI solvers. This paper will follow the line of [21] and will try to improve the existing results.

In this paper, we consider the problems of stability bound analysis and design of SPSs with time-varying delay. One contribution of this paper is that through the use of a LyapunovKrasovskii functional, the obtained results have turned out to be with less computational cost than the existing methods. Another contribution is that the proposed methods do not depend on the system decomposition and thus they can be applied to both standard and nonstandard SPSs with timevarying delay. The presented examples show that the obtained methods are of less computational cost than some existing ones, which results from newly developed LyapunovKrasovskii functionals.

\section{Main Results}

Consider the following SPSs with tine-varying delay:

$$
\begin{gathered}
E(\varepsilon) \dot{x}(t)=A x(t)+D x(t-d(t))+B u(t), \\
x(t)=\phi(t), \quad t \in[-\tau, 0),
\end{gathered}
$$

where $x(t) \in R^{n}$ is the system state, and $u(t) \in R^{m}$ is the control input. $\phi(t)$ is initial condition of the system. $E(\varepsilon)=$ $\left[\begin{array}{cc}I_{n_{1}} & 0 \\ 0 & \varepsilon I_{n_{2}}\end{array}\right] \in R^{n \times n}, A \in R^{n \times n}, D \in R^{n \times n}$, and $B \in R^{n \times m}$ are known constant matrices. $d(t)$ is the time-varying delay and satisfies

$$
0 \leq d(t) \leq \tau, \quad \dot{d}(t) \leq \mu<1,
$$

where $\tau$ and $\mu$ are known constants.

Condition (2) is common in practice and is widely used in the investigations on analysis and design of systems subject to time-varying delays [24-26]. In this paper, we aim at providing stability bound analysis and design method for system (1). Some useful lemmas are provided for subsequent technical development of the paper.

Lemma 1 (see [1]). Given $\bar{\varepsilon}>0$, symmetric matrices $S_{1}, S_{2}$, and $S_{3}$, if

$$
\begin{gathered}
S_{1} \geq 0, \\
S_{1}+\bar{\varepsilon} S_{2}>0, \\
S_{1}+\bar{\varepsilon} S_{2}+\bar{\varepsilon}^{2} S_{3}>0,
\end{gathered}
$$

hold, then

$$
S_{1}+\varepsilon S_{2}+\varepsilon^{2} S_{3}>0, \quad \forall \varepsilon \in(0, \bar{\varepsilon}] .
$$

Lemma 2 (see [1]). If there exist matrices $Z_{i}(i=1,2, \ldots, 5)$ of appropriate dimensions with $Z_{i}=Z_{i}^{T}(i=1,2,3,4)$ satisfying the following LMIs:

$$
\begin{gathered}
Z_{1}>0 \\
{\left[\begin{array}{cc}
Z_{1}+\bar{\varepsilon} Z_{3} & \bar{\varepsilon} Z_{5}^{T} \\
\bar{\varepsilon} Z_{5} & \bar{\varepsilon} Z_{2}
\end{array}\right]>0} \\
{\left[\begin{array}{cc}
Z_{1}+\bar{\varepsilon} Z_{3} & \bar{\varepsilon} Z_{5}^{T} \\
\bar{\varepsilon} Z_{5} & \bar{\varepsilon} Z_{2}+\bar{\varepsilon}^{2} Z_{4}
\end{array}\right]>0}
\end{gathered}
$$

then

$$
E(\varepsilon) Z(\varepsilon)=(E(\varepsilon) Z(\varepsilon))^{T}=Z^{T}(\varepsilon) E(\varepsilon)>0, \quad \forall \varepsilon \in(0, \bar{\varepsilon}],
$$

where

$$
Z(\varepsilon)=\left[\begin{array}{cc}
Z_{1}+\varepsilon Z_{3} & \varepsilon Z_{5}^{T} \\
Z_{5} & Z_{2}+\varepsilon Z_{4}
\end{array}\right], \quad E(\varepsilon)=\left[\begin{array}{cc}
I & 0 \\
0 & \varepsilon I
\end{array}\right] .
$$

2.1. Stability Analysis. Consider the following SPS with timevarying delay:

$$
\begin{gathered}
E(\varepsilon) \dot{x}(t)=A x(t)+D x(t-d(t)), \\
x(t)=\phi(t), \quad t \in[-\tau, 0) .
\end{gathered}
$$

Theorem 3. Given $\bar{\varepsilon}>0$, system (8) is asymptotically stable for $\forall \varepsilon \in(0, \bar{\varepsilon}]$, and any $d(t)$ satisfying $(2)$, if there exist symmetric positive-definite matrices $Q>0, M>0$, and matrices $Z_{i}(i=$ $1, \ldots, 5)$ with $Z_{i}=Z_{i}^{T}(i=1,2,3,4)$ satisfying the following LMIs:

$$
\begin{gathered}
Z_{1}>0, \\
{\left[\begin{array}{cc}
Z_{1}+\bar{\varepsilon} Z_{3} & \bar{\varepsilon} Z_{5}^{T} \\
\bar{\varepsilon} Z_{5} & \bar{\varepsilon} Z_{2}
\end{array}\right]>0,} \\
{\left[\begin{array}{cc}
Z_{1}+\bar{\varepsilon} Z_{3} & \bar{\varepsilon} Z_{5}^{T} \\
\bar{\varepsilon} Z_{5} & \bar{\varepsilon} Z_{2}+\bar{\varepsilon}^{2} Z_{4}
\end{array}\right]>0,} \\
{\left[\begin{array}{cc}
A^{T} Z(0)+Z^{T}(0) A+Q+\tau A^{T} M A & Z^{T}(0) D+\tau A^{T} M D \\
* & -(1-\mu) Q+\tau D^{T} M D
\end{array}\right]} \\
{\left[\begin{array}{cc}
A^{T} Z(\bar{\varepsilon})+Z^{T}(\bar{\varepsilon}) A+Q+\tau A^{T} M A & Z^{T}(\bar{\varepsilon}) D+\tau A^{T} M D \\
< &
\end{array}\right]} \\
<0,
\end{gathered}
$$

where $Z(\varepsilon)=\left[\begin{array}{cc}Z_{1}+\varepsilon Z_{3} & \varepsilon Z_{5}^{T} \\ Z_{5} & Z_{2}+\varepsilon Z_{4}\end{array}\right]$ 
Proof. Define a quadratic Lyapunov-Krasovskii functional as follows:

$$
\begin{aligned}
V\left(x_{t}\right)= & x^{T}(t) E(\varepsilon) Z(\varepsilon) x(t) \\
& +\int_{t-d(t)}^{t} x^{T}(s) Q x(s) d s \\
& +\int_{-\tau}^{0} \int_{t-d(t)+\theta}^{t}(E(\varepsilon) \dot{x}(s))^{T} M E(\varepsilon) \dot{x}(s) d s d \theta,
\end{aligned}
$$

where $Q>0, M>0$.

By Lemma 2, LMIs (9) imply that

$$
E(\varepsilon) Z(\varepsilon)=Z^{T}(\varepsilon) E(\varepsilon)>0, \quad \forall \varepsilon \in(0, \bar{\varepsilon}] .
$$

Thus $V\left(x_{t}\right)$ is a positive definite Lyapunov-Krasovskii functional.

By Lemma 1, it follows from (10) that

$$
\left[\begin{array}{cc}
A^{T} Z(\varepsilon)+Z^{T}(\varepsilon) A+Q+\tau A^{T} M A & Z^{T}(\varepsilon) D+\tau A^{T} M D \\
* & -(1-\mu) Q+\tau D^{T} M D
\end{array}\right]
$$

$<0$.

Taking the derivative of $V\left(x_{t}\right)$ along the trajectories of the system (8), we have

$$
\begin{aligned}
& \left.\dot{V}\left(x_{t}\right)\right|_{(8)} \\
& =\frac{d}{d t}\left(x^{T}(t) E(\varepsilon) Z(\varepsilon) x(t)\right) \\
& \quad+\frac{d}{d t}\left(\int_{t-d(t)}^{t} x^{T}(s) Q x(s) d s\right) \\
& +\frac{d}{d t}\left(\int_{-\tau}^{0} \int_{t-d(t)+\theta}^{t}(E(\varepsilon) \dot{x}(s))^{T} M E(\varepsilon) \dot{x}(s) d s d \theta\right) .
\end{aligned}
$$

It is easy to show that

$$
\begin{aligned}
\frac{d}{d t}( & \left.x^{T}(t) E(\varepsilon) Z(\varepsilon) x(t)\right) \\
= & \frac{d}{d t}\left(x^{T}(t) E(\varepsilon)\right) Z(\varepsilon) x(t) \\
& +x^{T}(t) E(\varepsilon) \frac{d}{d t}(Z(\varepsilon) x(t)) \\
= & \dot{x}^{T}(t) E(\varepsilon) Z(\varepsilon) x(t) \\
& +x^{T}(t) E(\varepsilon) Z(\varepsilon) \dot{x}(t) \\
= & (E(\varepsilon) \dot{x}(t))^{T} Z(\varepsilon) x(t) \\
& +x^{T}(t) Z^{T}(\varepsilon)(E(\varepsilon) \dot{x}(t)) \\
= & (A x(t)+D x(t-d(t)))^{T} Z(\varepsilon) x(t) \\
& +x^{T}(t) Z^{T}(\varepsilon)(A x(t)+D x(t-d(t)))
\end{aligned}
$$

$$
\begin{aligned}
= & x^{T}(t)\left(A^{T} Z(\varepsilon)+Z^{T}(\varepsilon) A\right) x(t) \\
& +(D x(t-d(t)))^{T} Z(\varepsilon) x(t) \\
& +(Z(\varepsilon) x(t))^{T} D x(t-d(t)) \\
= & x^{T}(t)\left(A^{T} Z(\varepsilon)+Z^{T}(\varepsilon) A\right) x(t) \\
& +2 x^{T}(t) Z^{T}(\varepsilon) D x(t-d(t)),
\end{aligned}
$$$$
\frac{d}{d t}\left(\int_{t-d(t)}^{t} x^{T}(s) Q x(s) d s\right)
$$$$
=x^{T}(t) Q x(t)-(1-\dot{d}(t)) x^{T}
$$$$
\times(t-d(t)) Q x(t-d(t))
$$$$
\leq x^{T}(t) Q x(t)-(1-\mu) x^{T}
$$$$
\times(t-d(t)) Q x(t-d(t)),
$$$$
\frac{d}{d t}\left(\int_{-\tau}^{0} \int_{t-d(t)+\theta}^{t}(E(\varepsilon) \dot{x}(s))^{T} M E(\varepsilon) \dot{x}(s) d s d \theta\right)
$$$$
=\tau(E(\varepsilon) \dot{x}(t))^{T} M E(\varepsilon) \dot{x}(t)-(1-\dot{d}(t))
$$

$$
\begin{aligned}
\times \int_{-\tau}^{0}(E(\varepsilon) \dot{x}(t-d(t)+\theta))^{T} \\
\times M E(\varepsilon) \dot{x}(t-d(t)+\theta) d \theta \\
\leq \tau(E(\varepsilon) \dot{x}(t))^{T} M E(\varepsilon) \dot{x}(t)-(1-\mu) \\
\times \int_{-\tau}^{0}(E(\varepsilon) \dot{x}(t-d(t)+\theta))^{T} \\
\quad \times M E(\varepsilon) \dot{x}(t-d(t)+\theta) d \theta .
\end{aligned}
$$

Since $M>0$, we have

$$
\begin{gathered}
\int_{-\tau}^{0}(E(\varepsilon) \dot{x}(t-d(t)+\theta))^{T} M E(\varepsilon) \dot{x}(t-d(t)+\theta) d \theta>0, \\
\frac{d}{d t}\left(\int_{-\tau}^{0} \int_{t-d(t)+\theta}^{t}(E(\varepsilon) \dot{x}(s))^{T} M E(\varepsilon) \dot{x}(s) d s d \theta\right) \\
\leq \tau(E(\varepsilon) \dot{x}(t))^{T} M E(\varepsilon) \dot{x}(t) .
\end{gathered}
$$

Therefore,

$$
\begin{aligned}
\left.\dot{V}\left(x_{t}\right)\right|_{(8)} \leq & x^{T}(t)\left(A^{T} Z(\varepsilon)+Z^{T}(\varepsilon) A\right) x(t) \\
& +2 x^{T}(t) Z^{T}(\varepsilon) D x(t-d(t)) \\
& +x^{T}(t) Q x(t)-(1-\mu) x^{T} \\
& \times(t-d(t)) Q x(t-d(t)) \\
& +\tau(E(\varepsilon) \dot{x}(t))^{T} M E(\varepsilon) \dot{x}(t) \\
= & \xi^{T}(t) H(\varepsilon) \xi(t),
\end{aligned}
$$


where $\xi(t)=\left(x^{T}(t) x^{T}(t-d)\right)^{T}$,

$H(\varepsilon)$

$=\left[\begin{array}{cc}A^{T} Z(\varepsilon)+Z^{T}(\varepsilon) A+Q+\tau A^{T} M A & Z^{T}(\varepsilon) D+\tau A^{T} M D \\ * & -(1-\mu) Q+\tau D^{T} M D\end{array}\right]$.

From (13), we get $H(\varepsilon)<0$, which yields

$$
\xi^{T}(t) H(\varepsilon) \xi(t)<0 .
$$

Therefore, $\left.\dot{V}\left(x_{t}\right)\right|_{(8)}<0$.

Hence, system $(8)$ is asymptotically stable for all $\varepsilon \in(0, \bar{\varepsilon}]$.

Remark 4. By Theorem 3, an estimate of the stability bound can be obtained. However, the results in [16-18, 22] are only sufficient conditions for the system to be stable for small enough singular perturbation parameter. holds.

By Theorem 3, it is easy to see that the following corollary

Corollary 5. Given $\bar{\varepsilon}>0$, system (8) is asymptotically stable for $\forall \varepsilon \in(0, \bar{\varepsilon}]$ and any $d(t)$ satisfying $d(t) \geq 0$ and $\dot{d}(t) \leq$ $\mu<1$, if there exist symmetric positive-definite matrices $Q>0$, and matrices $Z_{i}(i=1, \ldots, 5)$ with $Z_{i}=Z_{i}^{T}(i=1,2,3,4)$ satisfying the following LMIs:

$$
\begin{gathered}
Z_{1}>0 \\
{\left[\begin{array}{cc}
Z_{1}+\bar{\varepsilon} Z_{3} & \bar{\varepsilon} Z_{5}^{T} \\
\bar{\varepsilon} Z_{5} & \bar{\varepsilon} Z_{2}
\end{array}\right]>0} \\
{\left[\begin{array}{cc}
Z_{1}+\bar{\varepsilon} Z_{3} & \bar{\varepsilon} Z_{5}^{T} \\
\bar{\varepsilon} Z_{5} & \bar{\varepsilon} Z_{2}+\bar{\varepsilon}^{2} Z_{4}
\end{array}\right]>0} \\
{\left[\begin{array}{cc}
A^{T} Z(0)+Z^{T}(0) A+Q & Z^{T}(0) D \\
* & -(1-\mu) Q
\end{array}\right]<0,} \\
{\left[\begin{array}{cc}
A^{T} Z(\bar{\varepsilon})+Z^{T}(\bar{\varepsilon}) A+Q & Z^{T}(\bar{\varepsilon}) D \\
* & -(1-\mu) Q
\end{array}\right]<0}
\end{gathered}
$$

where $Z(\varepsilon)=\left[\begin{array}{cc}Z_{1}+\varepsilon Z_{3} & \varepsilon Z_{5}^{T} \\ Z_{5} & Z_{2}+\varepsilon Z_{4}\end{array}\right]$

Remark 6. In [21], stability bound problem was considered for SPSs with time delay, and LMI-based results were proposed. A new Lyapunov-Krasovskii functional is used to derive the analysis method. It is known that computational cost of LMI conditions increases in direct proportion to the number of decision variables and lines of the LMI conditions. In this sense, when the results of [21] are specialized to study stability of system (1), they need more computational cost than Theorem 3 and Corollary 5 of the present paper as will be illustrated by an example in the next section. In addition, the proposed Lyapunov-Krasovskii functional is expected to be used to study the problems of robust stability and $H_{\infty}$ control for singularly perturbed systems with time-varying delay.
Remark 7. When $\varepsilon=0$, system (8) becomes a singular system $[27,28]$. Stability of singular systems with time-varying delay has been considered by many researchers (see, e.g., [4]). Theorem 3 and Corollary 5 present sufficient conditions for system (8) to be stable when $\varepsilon \in(0, \bar{\varepsilon}]$, which excludes the case $\varepsilon=0$. In the future, we will study the stability problem of system (8) for $\varepsilon \in[0, \bar{\varepsilon}]$, which is also an interesting problem.

2.2. State Feedback Controller Design. In this subsection, we will design a state feedback controller for system (1) to achieve a given stability bound. A state feedback controller under consideration is in the form of

$$
u(t)=K x(t)
$$

where $K \in R^{m \times n}$ is the controller gain to be designed. Then the closed-loop system is as follows:

$$
E(\varepsilon) \dot{x}(t)=(A+B K) x(t)+D x(t-d(t)) .
$$

Theorem 8. Given $\bar{\varepsilon}>0$, if there exist matrices $Q>0, \widetilde{K}$ and $Z_{i}(i=1,2, \ldots, 5)$ with $Z_{i}=Z_{i}^{T}(i=1,2,3,4)$, satisfying the following LMIs:

$$
\begin{gathered}
Z_{1}>0 \\
{\left[\begin{array}{cc}
Z_{1}+\bar{\varepsilon} Z_{3} & \bar{\varepsilon} Z_{5}^{T} \\
\bar{\varepsilon} Z_{5} & \bar{\varepsilon} Z_{2}
\end{array}\right]>0} \\
{\left[\begin{array}{cc}
Z_{1}+\bar{\varepsilon} Z_{3} & \bar{\varepsilon} Z_{5}^{T} \\
\bar{\varepsilon} Z_{5} & \bar{\varepsilon} Z_{2}+\bar{\varepsilon}^{2} Z_{4}
\end{array}\right]>0} \\
{\left[\begin{array}{ccc}
A Z(0)+B \widetilde{K}+Z^{T}(0) A^{T}+\widetilde{K} B^{T}+Q & D Z(0) & Z^{T}(0) A^{T}+\widetilde{K} B^{T} \\
* & -(1-\mu) Q & Z^{T}(0) D^{T} \\
* & * & -\tau^{-1} \widetilde{M}
\end{array}\right]<0,} \\
{\left[\begin{array}{ccc}
A Z(\bar{\varepsilon})+B \widetilde{K}+Z^{T}(\bar{\varepsilon}) A^{T}+\widetilde{K} B^{T}+Q & D Z(\bar{\varepsilon}) & Z^{T}(\bar{\varepsilon}) A^{T}+\widetilde{K} B^{T} \\
* & -(1-\mu) Q & Z^{T}(\bar{\varepsilon}) D^{T} \\
* & * & -\tau^{-1} \widetilde{M}
\end{array}\right]<0,}
\end{gathered}
$$

where $Z(\varepsilon)=\left[\begin{array}{cc}Z_{1}+\varepsilon Z_{3} & \varepsilon Z_{5}^{T} \\ Z_{5} & Z_{2}+\varepsilon Z_{4}\end{array}\right]$, then the closed-loop system (22) is asymptotically stable for $\forall \varepsilon \in(0, \bar{\varepsilon}]$. Under such conditions, the gain matrix of controller (21) is $K=\widetilde{K} Z^{-1}(\varepsilon)$.

Proof. Choose the following Lyapunov functional candidate:

$$
\begin{aligned}
V\left(x_{t}\right)= & x^{T}(t) Z^{-T}(\varepsilon) E(\varepsilon) x(t) \\
& +\int_{t-d(t)}^{t} x^{T}(s) Z^{-T}(\varepsilon) Q Z^{-1}(\varepsilon) x(s) d s \\
& +\int_{-\tau}^{0} \int_{t-d(t)+\theta}^{t}(E(\varepsilon) \dot{x}(s))^{T} M E(\varepsilon) \dot{x}(s) d s d \theta,
\end{aligned}
$$

where $Q>0, M>0$. 
Now, considering the derivative of the Lyapunov functional candidate along the solution of SPSs with respect to $t$, we obtain

$$
\begin{aligned}
\dot{V}\left(x_{t}\right) & \left.\right|_{(22)} \\
= & \frac{d}{d t}\left(x^{T}(t) Z^{-T}(\varepsilon) E(\varepsilon) x(t)\right) \\
& +\frac{d}{d t}\left(\int_{t-d(t)}^{t} x^{T}(s) Z^{-T}(\varepsilon) Q Z^{-1}(\varepsilon) x(s) d s\right) \\
& +\frac{d}{d t}\left(\int_{-\tau}^{0} \int_{t-d(t)+\theta}^{t}(E(\varepsilon) \dot{x}(s))^{T} M E(\varepsilon) \dot{x}(s) d s d \theta\right) .
\end{aligned}
$$

By Lemma 2, LMIs (23), (24), and (25) imply that

$$
E(\varepsilon) Z(\varepsilon)=(E(\varepsilon) Z(\varepsilon))^{T}=Z^{T}(\varepsilon) E(\varepsilon),
$$

which shows

$$
Z^{-T}(\varepsilon) E(\varepsilon) Z(\varepsilon)=Z^{-T}(\varepsilon) Z^{T}(\varepsilon) E(\varepsilon)=E(\varepsilon) .
$$

Then

$$
Z^{-T}(\varepsilon) E(\varepsilon)=E(\varepsilon) Z^{-1}(\varepsilon) .
$$

Consequently,

$$
\begin{aligned}
\frac{d}{d t} & \left(x^{T}(t) Z^{-T}(\varepsilon) E(\varepsilon) x(t)\right) \\
= & \dot{x}^{T}(t) Z^{-1}(\varepsilon) E(\varepsilon) x(t)+x^{T}(t) Z^{-T}(\varepsilon) E(\varepsilon) \dot{x}(t) \\
= & \dot{x}^{T}(t) E(\varepsilon) Z^{-1}(\varepsilon) x(t)+\left(Z^{-1}(\varepsilon) x(t)\right)^{T} E(\varepsilon) \dot{x}(t) \\
= & 2\left(Z^{-1}(\varepsilon) x(t)\right)^{T} E(\varepsilon) \dot{x}(t) \\
= & 2\left(Z^{-1}(\varepsilon) x(t)\right)^{T}[(A+B K) x(t)+D x(t-d(t))] \\
= & 2 x^{T}(t) Z^{-T}(\varepsilon)(A+B K) x(t) \\
& +2 x^{T}(t) Z^{-T}(\varepsilon) D x(t-d(t)), \\
\frac{d}{d t}( & \left.\int_{t-d(t)}^{t} x^{T}(s) Z^{-T}(\varepsilon) Q Z^{-1}(\varepsilon) x(s) d s\right) \\
= & x^{T}(t) Z^{-T}(t) Q Z^{-1}(t) x(t) \\
& -(1-\dot{d}(t)) x^{T}(t-d(t)) Z^{-T}(t-d(t)) \\
& \times Q Z^{-1}(t-d(t)) x(t-d(t)) \\
\leq & x^{T}(t) Z^{-T}(t) Q Z^{-1}(t) x(t) \\
& -(1-\mu) x^{T}(t-d(t)) Z^{-T}(t-d(t)) \\
& \times Q Z^{-1}(t-d(t)) x(t-d(t)),
\end{aligned}
$$

$$
\begin{aligned}
\frac{d}{d t}\left(\int_{-\tau}^{0} \int_{t-d(t)+\theta}^{t}(E(\varepsilon) \dot{x}(s))^{T} M E(\varepsilon) \dot{x}(s) d s d \theta\right) \\
=\tau(E(\varepsilon) \dot{x}(t))^{T} M E(\varepsilon) \dot{x}(t) \\
\quad-(1-\dot{d}(t)) \int_{-\tau}^{0}(E(\varepsilon) \dot{x}(t-d(t)+\theta))^{T} M E(\varepsilon) \\
\quad \times \dot{x}(t-d(t)+\theta) d \theta \\
\leq \tau(E(\varepsilon) \dot{x}(t))^{T} M E(\varepsilon) \dot{x}(t) \\
\quad-(1-\mu) \int_{-\tau}^{0}(E(\varepsilon) \dot{x}(t-d(t)+\theta))^{T} M E(\varepsilon) \\
\leq \\
=\tau(E(\varepsilon) \dot{x}(t))^{T} M E(\varepsilon) \dot{x}(t) \\
\quad+(A+B K) x(t)+D x(t-d(t))]^{T} \\
\quad \times M[(A+B K) x(t)+D x(t-d(t))] \\
=\tau x^{T}(t)(A+B K)^{T} M(A+B K) x(t) \\
+\tau 2 x^{T}(t)(A+B K)^{T} M D x^{T}(t-d(t)) \\
\quad d(t)) D^{T} M D x(t-d(t)) .
\end{aligned}
$$

Therefore, taking the derivative of $V\left(x_{t}\right)$ along the trajectories of the system (22), we have

$$
\begin{aligned}
\left.\dot{V}\left(x_{t}\right)\right|_{(22)} \leq & 2 x^{T}(t) Z^{-T}(\varepsilon)(A+B K) x(t) \\
& +2 x^{T}(t) Z^{-T}(\varepsilon) D x(t-d(t)) \\
& -(1-\mu) x^{T}(t-d(t)) Z^{-T}(t-d(t)) \\
& \times Q Z^{-1}(t-d(t)) x(t-d(t)) \\
& +x^{T}(t) Z^{-T}(t) Q Z^{-1}(t) x(t) \\
& +\tau x^{T}(t)(A+B K)^{T} M(A+B K) x(t) \\
& +\tau 2 x^{T}(t)(A+B K)^{T} M D x^{T}(t-d(t)) \\
& +\tau x^{T}(t-d(t)) D^{T} M D x(t-d(t)) \\
= & \xi^{T}(t) G(\varepsilon) \xi(t),
\end{aligned}
$$

where $\xi(t)=\left(x^{T}(t) x^{T}(t-d)\right)^{T}$,

$$
G(\varepsilon)=\left[\begin{array}{cc}
\Xi & Z^{-T}(\varepsilon) D+\tau(A+B K)^{T} M D \\
* & \Pi
\end{array}\right],
$$

with

$$
\begin{aligned}
& \Xi= Z^{-T}(\varepsilon)(A+B K)+(A+B K)^{T} Z^{-1}(\varepsilon) \\
&+\tau(A+B K)^{T} M(A+B K)+Z^{-T}(\varepsilon) Q Z^{-1}(\varepsilon), \\
& \Pi=-(1-\mu) Z^{-T}(\varepsilon) Q Z^{-1}(\varepsilon)+\tau D^{T} M D .
\end{aligned}
$$


By defining

$$
Y=\left[\begin{array}{cc}
Z^{-T}(\varepsilon)(A+B K)+(A+B K)^{T} Z^{-1}(\varepsilon)+Z^{-T}(\varepsilon) Q Z^{-1}(\varepsilon) & Z^{-T}(\varepsilon) D \\
* & -(1-\mu) Z^{-T}(\varepsilon) Q Z^{-1}(\varepsilon)
\end{array}\right]
$$

algebraic manipulation gives

$$
\begin{aligned}
G(\varepsilon) & =Y+\left[\begin{array}{cc}
\tau(A+B K)^{T} M(A+B K) & \tau(A+B K)^{T} M D \\
* & \tau D^{T} M D
\end{array}\right] \\
& =Y+\left[\begin{array}{cc}
\tau(A+B K)^{T} M(A+B K) & \tau(A+B K)^{T} M D \\
* & \tau D^{T} M D
\end{array}\right] \\
& =Y+\left[\begin{array}{c}
(A+B K)^{T} \\
D^{T}
\end{array}\right] \tau M\left[\begin{array}{ll}
A+B K & D
\end{array}\right] \\
& =Y-\left[\begin{array}{c}
(A+B K)^{T} \\
D^{T}
\end{array}\right]\left(-\tau^{-1} M^{-1}\right)^{-1}\left[\begin{array}{ll}
A+B K & D
\end{array}\right] .
\end{aligned}
$$

Now comes the validation that $G(\varepsilon)<0$, by the Schur complement, which is equivalent to

$$
\left[\begin{array}{ccc}
\Theta & Z^{-T}(\varepsilon) D & (A+B K)^{T} \\
* & -(1-\mu) Z^{-T}(\varepsilon) Q Z^{-1}(\varepsilon) & D^{T} \\
* & * & -\tau^{-1} M^{-1}
\end{array}\right]<0,
$$

where $\Theta=Z^{-T}(\varepsilon)(A+B K)+(A+B K)^{T} Z^{-1}(\varepsilon)+Z^{-T}(\varepsilon)$ $\mathrm{QZ}^{-1}(\varepsilon)$.

Pre- and postmultiplying (38) with $\operatorname{diag}\left\{Z^{T}(\varepsilon), Z^{T}(\varepsilon), I\right\}$ and its transpose, respectively, we obtain

$$
\begin{aligned}
& {\left[\begin{array}{ccc}
(A+B K) Z(\varepsilon)+[(A+B K) Z(\varepsilon)]^{T}+Q & D Z(\varepsilon) & {[(A+B K) Z(\varepsilon)]^{T}} \\
* & -(1-\mu) Q & Z^{T}(\varepsilon) D^{T} \\
* & * & -\tau^{-1} M^{-1}
\end{array}\right]} \\
& \quad=\left[\begin{array}{ccc}
A Z(\varepsilon)+B K Z(\varepsilon)+Z^{T}(\varepsilon) A^{T}+[B K Z(\varepsilon)]^{T}+Q & D Z(\varepsilon) & Z^{T}(\varepsilon) A^{T}+[B K Z(\varepsilon)]^{T} \\
* & -(1-\mu) Q & Z^{T}(\varepsilon) D^{T} \\
* & * & -\tau^{-1} M^{-1}
\end{array}\right]<0 .
\end{aligned}
$$

Defining

$$
\widetilde{K}=K Z(\varepsilon), \quad \widetilde{M}=M^{-1},
$$

we can obtain

$$
\widetilde{G}(\varepsilon)=\left[\begin{array}{ccc}
A Z(\varepsilon)+B \widetilde{K}+Z^{T}(\varepsilon) A^{T}+\widetilde{K} B^{T}+Q & D Z(\varepsilon) & Z^{T}(\varepsilon) A^{T}+\widetilde{K} B^{T} \\
* & -(1-\mu) Q & Z^{T}(\varepsilon) D^{T} \\
* & * & -\tau^{-1} \widetilde{M}
\end{array}\right]<0
$$

It follows from (26) that $\widetilde{G}(0)<0$ and $\widetilde{G}(\bar{\varepsilon})<0$. Then, by Lemma 1, we have that inequality (41) holds, that is, $\widetilde{G}(\varepsilon)<0$, which is equivalent to $G(\varepsilon)<0$. Then

$$
\left.\dot{V}\left(x_{t}\right)\right|_{(22)}<0 .
$$

This implies that the closed-loop system (22) is asymptotically stable for $\forall \varepsilon \in(0, \bar{\varepsilon}]$ with the gain matrix $K=\widetilde{K} Z^{-1}(\varepsilon)$. This completes the proof.
Remark 9. It follows from LMIs (23) and (24) that $Z_{1}$ and $Z_{2}$ are nonsingular matrices, which guarantees that $Z(0)$ is nonsingular. Then $K=\widetilde{K} Z^{-1}(\varepsilon)$ is well defined for $\forall \varepsilon \in(0, \bar{\varepsilon}]$.

Remark 10. In [20], the synthesis of SPSs with multiple time delays was considered. The proposed method depends on the separation of the original system into fast and slow subsystems and therefore cannot be applied to nonstandard SPSs. In [23], an LMI-based controller design method was proposed. But it did not consider the stability bound of the closedloop system. Using Theorem 8, a stabilization bound can be 
TABLE 1: Stability bounds of Example 11.

\begin{tabular}{lccccc}
\hline$\mu$ & 0 & 0.2 & 0.4 & 0.6 & 0.65 \\
$\bar{\varepsilon}$ & 0.4999 & 0.3924 & 0.2636 & 0.1027 & 0.0557 \\
\hline
\end{tabular}

TABLE 2: Comparison of the number of decision variables and lines of the LMI conditions.

\begin{tabular}{lcccc}
\hline & Theorem 3 & Theorem 3 of [21] & Corollary 5 & Theorem 6 in [21] \\
\hline Variables & 11 & 61 & 8 & 9 \\
Lines & 17 & 78 & 15 & 18 \\
\hline
\end{tabular}

achieved by the proposed state feedback controller. Moreover, it is easy to see that no system separation is required in our results and the conditions obtained in Theorem 8 have turned out to deal with both standard and nonstandard SPSs.

\section{Examples}

In this section, we provide two examples to demonstrate the validity and the advantage of the proposed results in this paper.

Example 11. Consider the following system with time-varying delay:

$$
\begin{gathered}
\dot{x}_{1}(t)=x_{2}(t)+x_{1}(t-d(t)), \\
\varepsilon \dot{x}_{2}(t)=-x_{2}(t)+0.5 x_{2}(t-d(t))-2 x_{1}(t) .
\end{gathered}
$$

This system can be transformed into system (8) with

$$
A=\left[\begin{array}{cc}
0 & 1 \\
-2 & -1
\end{array}\right], \quad D=\left[\begin{array}{cc}
1 & 0 \\
0 & 0.5
\end{array}\right]
$$

Solving the LMI conditions of Theorem 3 with $\bar{\varepsilon}=0.4482$, $\tau=0.55$, and $\mu=0.1$, we obtain

$$
\begin{gathered}
Z_{1}=3.5345, \quad Z_{2}=1.8589, \quad Z_{3}=4.0958, \\
Z_{4}=1.9564, \quad Z_{5}=2.9219, \\
Q=\left[\begin{array}{ll}
5.5894 & 1.3679 \\
1.3679 & 1.4026
\end{array}\right], \quad M=\left[\begin{array}{cc}
0.0120 & -0.0040 \\
-0.0040 & 0.0130
\end{array}\right] .
\end{gathered}
$$

Then by Theorem 3, the sys-
tem is stable for any delay satisfying $0 \leq d(t) \leq 5.5, \dot{d}(t) \leq 0.1$, and any $\varepsilon \in(0,0.4482]$. We found that the largest stability bound obtained by Theorem 3 of [21] is also 0.4482 when the involved time delay satisfies $0 \leq d(t) \leq 5.5, \dot{d}(t) \leq 0.1$.

When the time delay involved in this example satisfies $d(t) \geq 0$ and $\dot{d}(t) \leq \mu<1$, by Corollary 5 , the stability bounds for different $\mu$ are shown in Table 1 . It can be seen that the results are the same to those given by Theorem 6 in [21].

From Table 2, Theorem 3 and Corollary 5 in the present paper need less computational cost than Theorems 3 and 6 of [21], respectively. Therefore, it can be seen that the newly developed methods can produce the same stability bound with reduced computational cost than the existing method in [21].

Example 12. We now apply the proposed approach in Theorem 8 to find a state feedback controller to stabilize system (1) with

$$
A=\left[\begin{array}{cc}
1 & 1 \\
-2 & 0
\end{array}\right], \quad D=\left[\begin{array}{cc}
1 & 0 \\
0 & 0.5
\end{array}\right], \quad B=\left[\begin{array}{l}
0 \\
1
\end{array}\right]
$$

Letting $\tau=5, \mu=0.2$, and $\bar{\varepsilon}=0.4$ and using Theorem 8 , we obtain

$$
\begin{aligned}
& Z_{1}=0.0439, \quad Z_{2}=0.1801, \quad Z_{3}=0.0689, \\
& Z_{4}=0.0548, \quad Z_{5}=-0.1787, \\
& \widetilde{K}=\left[\begin{array}{ll}
0.1713 & -0.4163
\end{array}\right], \quad M=\left[\begin{array}{cc}
1.3566 & -0.3795 \\
-0.3795 & 2.1505
\end{array}\right] \text {, } \\
& Q=\left[\begin{array}{cc}
0.1119 & -0.0772 \\
-0.0772 & 0.2276
\end{array}\right]
\end{aligned}
$$

Then the controller gain is as follows:

$$
\begin{aligned}
K= & {\left[\begin{array}{ll}
0.1713 & -0.4163
\end{array}\right] } \\
& \times\left[\begin{array}{cc}
0.0439+0.0689 \varepsilon & -0.1787 \varepsilon \\
-0.1787 & 0.1801+0.0548 \varepsilon
\end{array}\right]^{-1} .
\end{aligned}
$$

By Theorem 8 , the closed-loop system is stable for any $0 \leq$ $d(t) \leq 5, \dot{d}(t) \leq 0.2$, and any $\varepsilon \in(0,0.4]$.

This example shows that Theorem 8 can be applied to nonstandard SPSs with time-varying delay.

\section{Conclusion}

In this paper, we have investigated the problems of stability bound analysis and design for singularly perturbed systems with time-varying delay. The results extend and improve the existing works. A new form of Lyapunov-Krasovskii functional has been constructed to improve delay-dependent stability analysis and design methods for SPSs with time-varying delay. Using the analysis methods, the stability bound of the 
SPSs can be computed. By the design methods, a given stability bound can be achieved. The proposed methods do not depend on the system decomposition and therefore can be applied to both standard and nonstandard SPSs with timevarying delays. The numerically examples have illustrated the advantages and effectiveness of the proposed methods.

\section{Acknowledgments}

This work was supported by the Natural Science Foundation of China (60904009, 60904079, 60974004, and 61020106003) and the Fundamental Research Funds for the Central Universities (N100406010 and N110408001).

\section{References}

[1] C. Yang and Q. Zhang, "Multiobjective control for T-S fuzzy singularly perturbed systems," IEEE Transactions on Fuzzy Systems, vol. 17, no. 1, pp. 104-115, 2009.

[2] H. Liu, F. Sun, K. He, and Z. Sun, "Survey of singularly perturbed control systems: theory and applications," Journal of Control Theory and Applications, vol. 20, no. 1, pp. 1-7, 2003.

[3] S. He, F. Da, and W. You, "Research advances of time-delay control theory," Journal of Nanjing University of Science and Technology, vol. 29, pp. 132-136, 2005.

[4] S. Cong and Z. Sheng, "On exponential stability conditions of descriptor systems with time-varying delay," Journal of Applied Mathematics, vol. 2012, Article ID 532912, 12 pages, 2012.

[5] M. da la Sen, "Quadratic stability and stabilization of switched dynamic systems with uncommensurate internal point delays," Applied Mathematics and Computation, vol. 185, no. 1, pp. 508526, 2007.

[6] V. Y. Glizer, "Euclidean space controllability of singularly perturbed linear systems with state delay," Systems \& Control Letters, vol. 43, no. 3, pp. 181-191, 2001.

[7] V. Y. Glizer, "Observability of singularly perturbed linear timedependent differential systems with small delay," Journal of Dynamical and Control Systems, vol. 10, no. 3, pp. 329-363, 2004.

[8] V. Y. Glizer, "Novel controllability conditions for a class of singularly-perturbed systems with small state delays," Journal of Optimization Theory and Applications, vol. 137, no. 1, pp. 135156, 2008.

[9] V. Y. Glizer and E. Fridman, "Stability of singularly perturbed functional-differential systems: spectrum analysis and LMI approaches," IMA Journal of Mathematical Control and Information, vol. 29, no. 1, pp. 79-111, 2012.

[10] V. Y. Glizer and E. Fridman, " $H_{\infty}$ control of linear singularly perturbed systems with small state delay," Journal of Mathematical Analysis and Applications, vol. 250, no. 1, pp. 49-85, 2000.

[11] D. W. Luse, "Multivariable singularly perturbed feedback systems with time delay," IEEE Transactions on Automatic Control, vol. 32, no. 11, pp. 990-994, 1987.

[12] Z. Shao and J. R. Rowland, "Stability of time-delay singularly perturbed systems," IEE Proceedings, vol. 142, no. 2, pp. 111-113, 1995.

[13] S. T. Pan, F. H. Hsiao, and C. C. Teng, "Stability bound of multiple time delay singularly perturbed systems," Electronics Letters, vol. 32, no. 14, pp. 1327-1328, 1996.

[14] Z. Shao, "Robust stability of singularly perturbed systems with state delays," IEE Proceedings, vol. 150, no. 1, pp. 2-6, 2003.
[15] S. B. Stojanovic and J. Debel, "Delay-dependent stability analysis for discrete-time systems with time varying state delay," Chemical Industry and Chemical Engineering Quarterly, vol. 17, pp. 497-504, 2011.

[16] L. L. Liu, J. P. Peng, and B. W. Wu, "Robust stability of singularly perturbed systems with state delays," in Proceedings of the International Workshop on Information Security and Application (IWISA '09), pp. 19-21, Qingdao, China, November 2009.

[17] E. Fridman, "Effects of small delays on stability of singularly perturbed systems," Automatica, vol. 38, no. 5, pp. 897-902, 2002.

[18] L. L. Liu, J. G. Peng, and B. W. Wu, "Delay-dependent criteria for robust stability of singularly perturbed systems with delays," in Proceedings of the International Conference on Computational and Information Sciences (ICCIS '10), pp. 1-4, December 2010.

[19] J.-S. Chiou, "Stability bound of discrete multiple time-delay singularly perturbed systems," International Journal of Systems Science, vol. 37, no. 14, pp. 1069-1076, 2006.

[20] P.-L. Liu, "Stabilization of singularly perturbed multiple-timedelay systems with a saturating actuator," International Journal of Systems Science, vol. 32, no. 8, pp. 1041-1045, 2001.

[21] W.-H. Chen, S.-T. Yang, X. Lu, and Y. Shen, "Exponential stability and exponential stabilization of singularly perturbed stochastic systems with time-varying delay," International Journal of Robust and Nonlinear Control, vol. 20, no. 18, pp. 20212044, 2010.

[22] P. Mei, C. X. Cai, and Y. Zou, "Stability analysis for singularly perturbed systems with time-varying delay," Journal of Nanjing University of Science and Technology, vol. 33, no. 3, pp. 297-301, 2009.

[23] Z. H. Shao, "Stability bounds of singularly perturbed delay systems," IEE Proceedings, vol. 151, pp. 585-588, 2004.

[24] J.-H. Kim, "Robust stability of linear systems with delayed perturbations," IEEE Transactions on Automatic Control, vol. 41, no. 12, pp. 1820-1822, 1996.

[25] P. Suthee and K. Furuta, "Memoryless stabilization of uncertain linear systems including time-varying state delays," IEEE Transactions on Automatic Control, vol. 34, pp. 460-462, 1989.

[26] Y.-Y. Cao and Y.-X. Sun, "Robust stabilization of uncertain systems with time-varying multistate delay," IEEE Transactions on Automatic Control, vol. 43, no. 10, pp. 1484-1488, 1998.

[27] M. De la Sen, "On positivity of singular regular linear timedelay time-invariant systems subject to multiple internal and external incommensurate point delays," Applied Mathematics and Computation, vol. 190, no. 1, pp. 382-401, 2007.

[28] S. L. Campbell, "A general form for solvable linear time varying singular systems of differential equations," SIAM Journal on Mathematical Analysis, vol. 18, no. 4, pp. 1101-1115, 1987. 


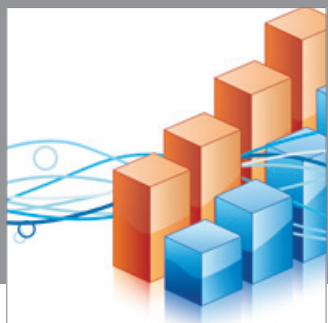

Advances in

Operations Research

mansans

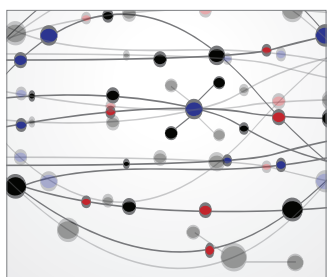

The Scientific World Journal
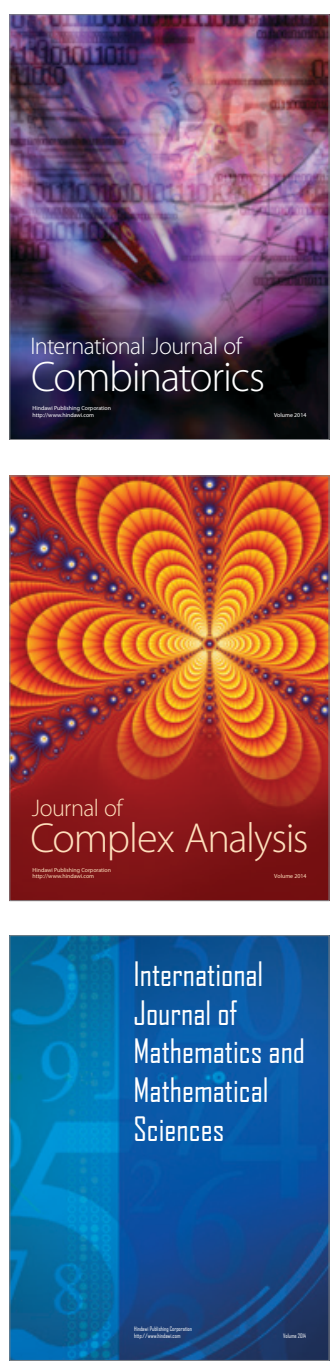
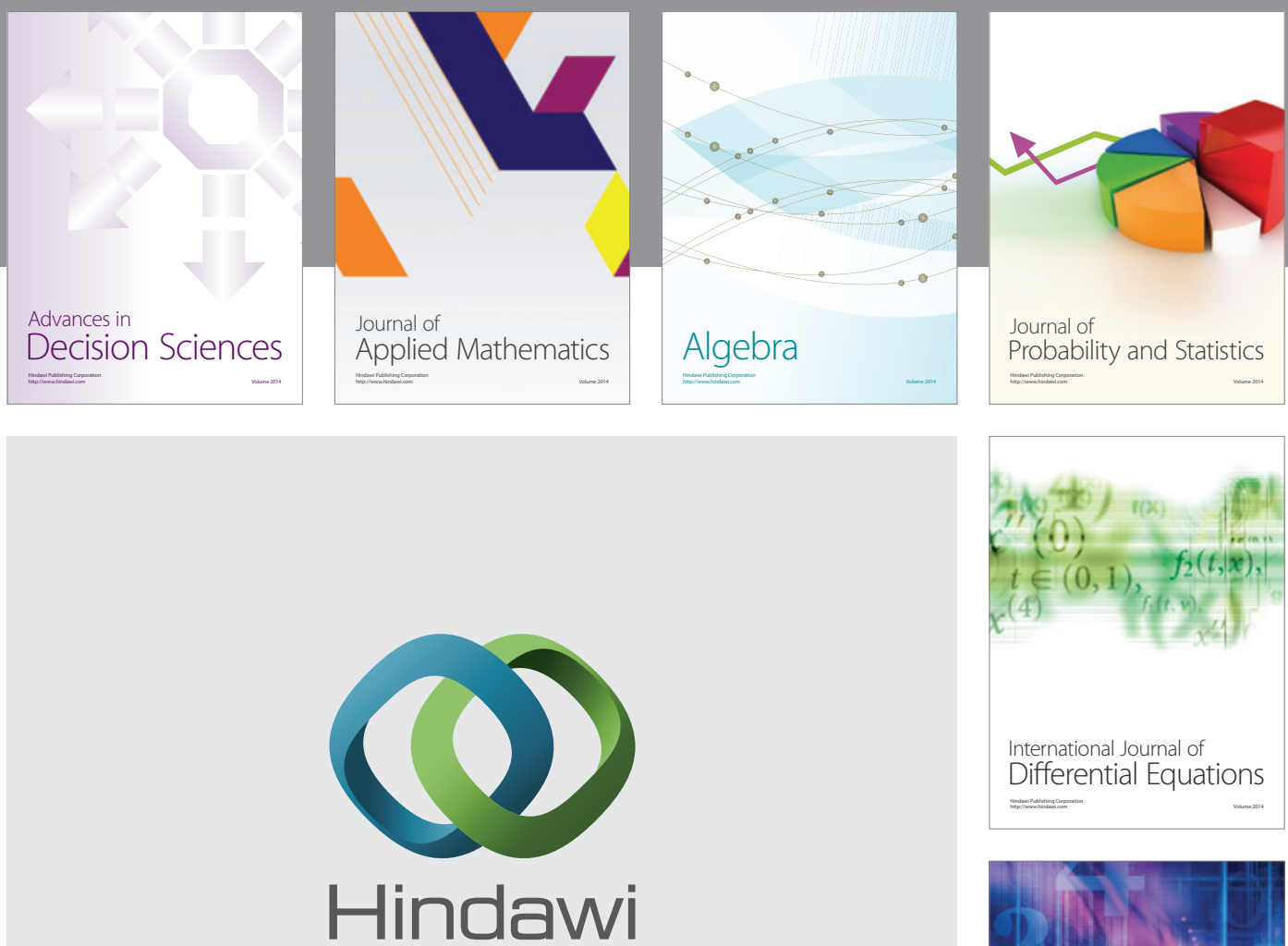

Submit your manuscripts at http://www.hindawi.com
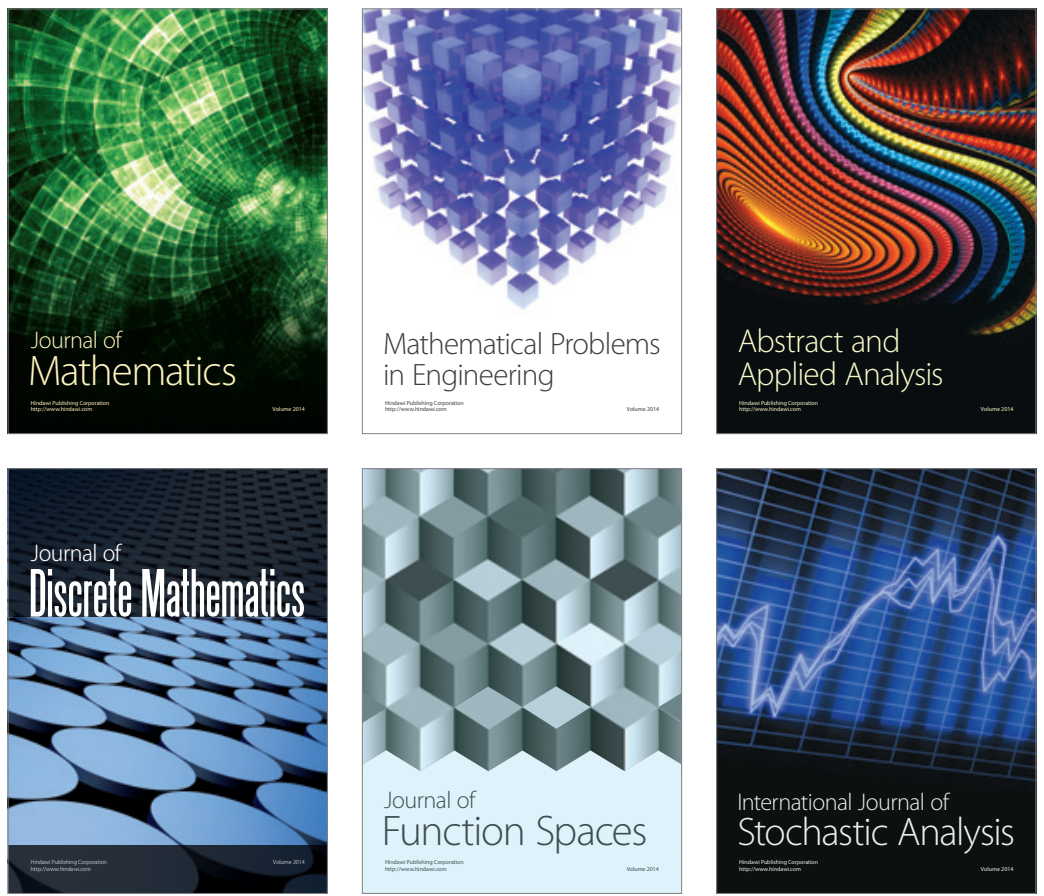

Journal of

Function Spaces

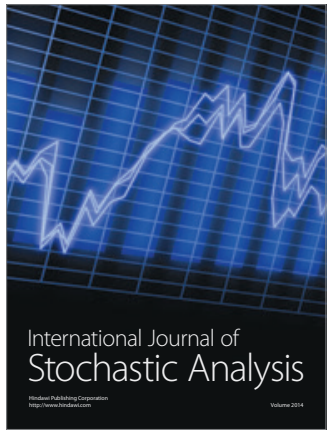

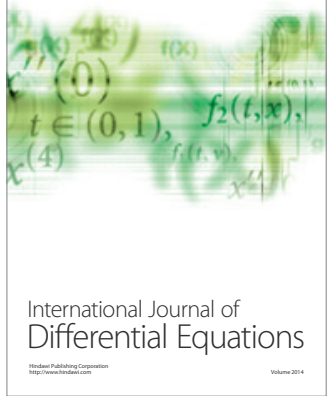
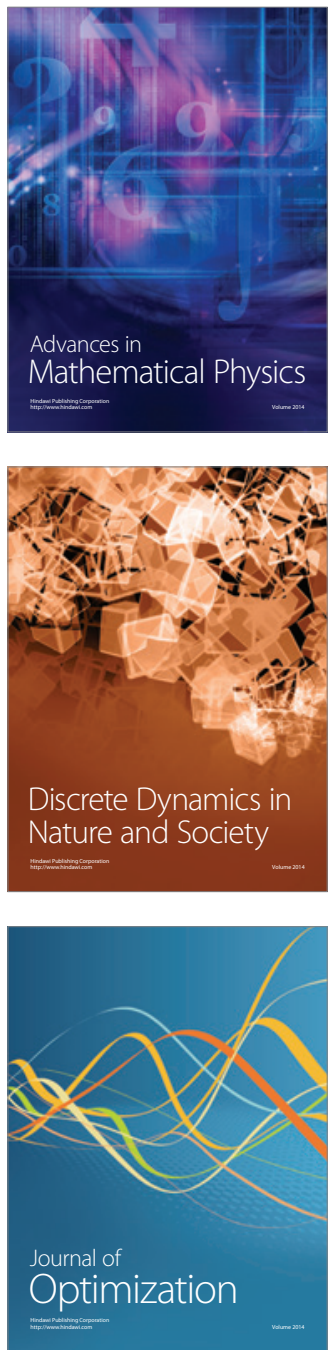